\title{
Applicability of partition approximation source locating tech- nique for a transversely isotropic medium
}

\author{
${ }^{1}$ Geology Division, Korea Institute of Geoscience and Mineral Resources, 124 Gwahak-ro, Yuseong-gu, Daejeon, 34132, Korea \\ ${ }^{2}$ Department of Energy Systems Engineering, Seoul National University, 1 Gwanka-ro, Gwanka-gu, Seoul, 08826, Korea; *Corresponding \\ author,E-mail:sjeon@snu.ac.kr
}

(Received: November 5, 2019; Revised accepted: April 28, 2020)

https://doi.org/10.18814/epiiugs/2020/020063

Microfracturing in a solid generates elastic waves, being detected in a form of acoustic emission (AE). Using an array of $A E$ sensors, the number and the location of $A E$ sources can be successfully monitored. Rocks usually show anisotropic mechanical, thermal, and hydraulic characteristics. Especially, many sedimentary rocks present transversely isotropic features originated from complex geological processes. If the anisotropy were not properly considered, the accuracy and precision of the AE source locating would not be guaranteed. In this study, one of the source locating techniques, called partition approximation method, was investigated to check its applicability to a transversely isotropic medium. To validate the technique quantitatively, analytic and experimental investigations were conducted. Ideal and virtual two-dimensional specimens were used to check it and as a result, the technique was capable of locating both isotropic and transversely isotropic cases, showing maximum $6.9 \%$ of error when normalized by the size of specimen. Also, pencil lead break tests on artificial transversely isotropic specimen were performed. The averaged error was measured as $7.46 \mathrm{~mm}$, which was smaller than the diameter of AE sensor used. In addition, Brazilian tension tests were conducted with $A E$ monitoring as an application of the technique.

\section{Introduction}

Various reasons, such as cracking, dislocation, and deformation of particles in a medium, can cause energy release and released energy is transformed into elastic waves that propagate through the medium. Acoustic emission (AE) is one type of the elastic waves that can be detected by piezoelectric sensors. As a non-destructive method of testing and/or monitoring, it is quite useful in detection of cracking behavior in brittle materials under various circumstances (Ouyang et al., 1991; Labuz et al., 2001; Soulioti et al., 2009; Ohno and Ohtsu, 2010). It has been extensively used to monitor and evaluate safety of civil structures, such as tunnels, bridges, rock slopes, and nuclear waste depositories (Olsson and Winberg, 1996; Kim et al., 2011; Huang et al., 2016). Furthermore, if multiple AE sensors are used, source locating and moment tensor analysis become available to examine the microscopic behavior of cracks (Ohtsu, 1995; Grosse et al., 1997).

Rocks usually show anisotropic mechanical, thermal, and hydraulic characteristics. In particular, rocks with planar structures, such as bedding plane and/or foliation, are well classified to be transversely isotropic. The transverse isotropy is a frequently adopted model in geomechanical modelling, especially for sedimentary rocks. Elastic wave velocities of these media vary by orientation according to the angle between the direction of isotropic plane and the direction of wave propagation (Daley and Hron, 1977; Song et al., 2004). Degree of variation depends on the nature of the medium.

Location of an $\mathrm{AE}$ event can be determined on the basis of elastic wave velocity through a medium and measured time differences between $\mathrm{AE}$ sensors. This process is called source locating (Ishida et al., 2017). Specific array of AE sensors allows less number of them in some occasion (Ebrahimkhanlou and Salamone, 2017; Yin et al., 2018), however, at least three $\mathrm{AE}$ sensors are required to locate an $\mathrm{AE}$ event in a two-dimensional case and at least four sensors in a threedimensional case normally. Several source locating techniques have been proposed, such as the least squares method, difference approximation method, table look-off method, and the partition approximation method (Ishida, 1999; Chang, 2002).

The former two techniques, i.e., least squares and difference approximation method, define an objective function based on the location of unknown AE source then, solve the problem by making residual of the function minimum (Scruby et al., 1985; Maji et al., 1990). In the case of isotropy, a constant wave velocity allows a simple function and corresponding simultaneous equation, but in the apparent anisotropic case, wave velocity results in nonlinear equations, which makes the calculation process complicated. Meanwhile, the other two techniques utilize a database of time differences. The wave anisotropy could be easily considered in the database so that simple calculations are required in the process even though the amount of calculation would be somewhat large.

In this study, the partition approximation method was adopted to take wave anisotropy into account owing to its brevity. Also, it has a 
relative strength against table look-off method in terms of calculation efficiency. In order to validate the applicability of the technique, analytic and numeric calculations were conducted. It showed the importance of selecting suitable technique, comparing the source locating results with and without considering the wave velocity anisotropy. At the same time, laboratory tests, especially pencil lead break tests, were performed so as to confirm the precision of the technique in a quantitative and practical manner.

Furthermore, a series of Brazilian tension tests were carried out to demonstrate the applicability of the partition approximation method. In the case of an isotropic specimen, tensile cracks are generated and propagated along the center line of the specimen, parallel to the direction of loading. However, cracking behavior in a transversely isotropic specimen greatly differs from that of an isotropic one due to the effect of anisotropy. Brazilian tension test was selected as an application, since the cracking behavior of the test is relatively well established and easy to be observed. In the experiments, AE sensors were attached onto the Brazilian disk to locate cracks and to investigate their cracking behavior. At the same time, a high speed camera was used to record the cracking behavior during the experiment and to compare the recordings with source locating results.

\section{Background}

\section{Partition Approximation Method}

Partition approximation and table look-off method divide an entire medium into many small elements and nodes, respectively. Supposing a rectangular partitioning in a two-dimensional case, the table

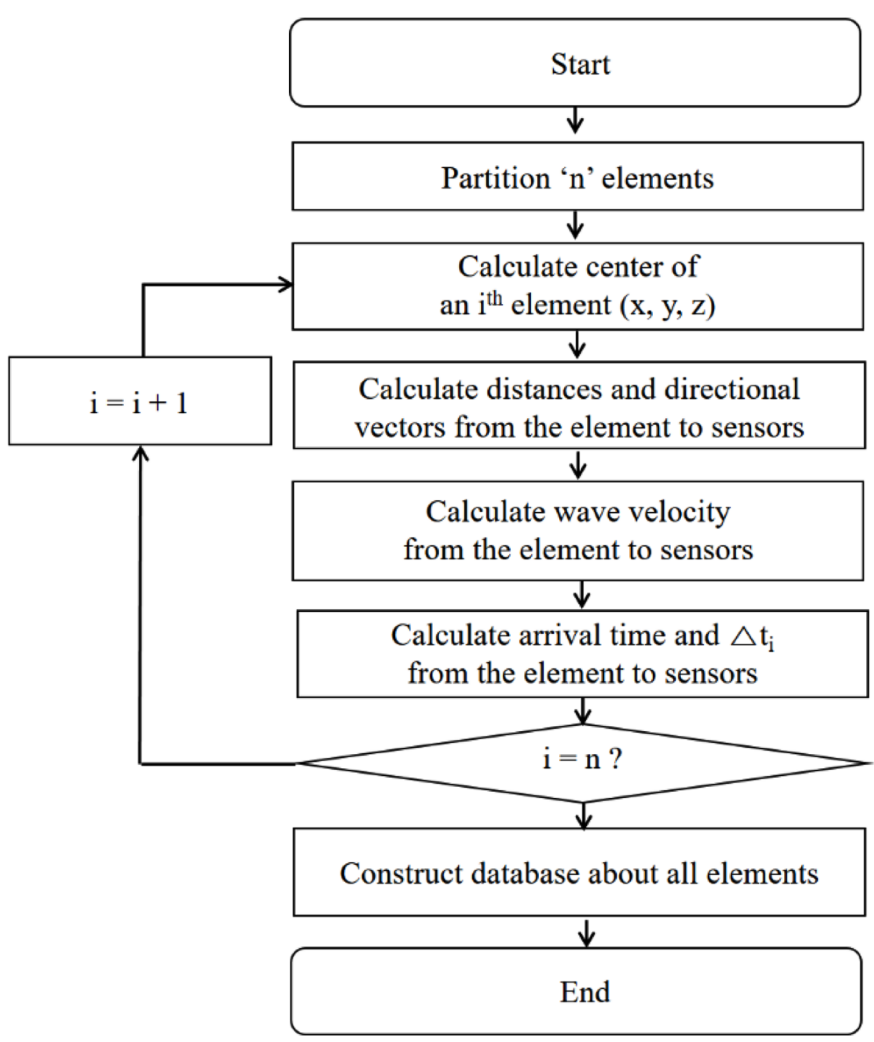

look-off method treats a node as a potential source, while the partition approximation method treats the center of element as a potential source. Therefore, partition approximation could secure better calculation efficiency and at the same time, discrepancies between those two techniques would not be significant if the medium were partitioned small enough.

Center of each element is treated as a potential source of $\mathrm{AE}$ at the early stage. Distance between each potential source and AE sensor can be calculated using coordinates of element and sensor, which are known parameters. Then, dividing the distance by the wave velocity gives the wave arrival time and arrival time differences between $\mathrm{AE}$ sensors. Therefore, a database of the arrival time differences $(\Delta t)$ can be constructed. By comparing experimental results with the database, an element with the least error is determined as the location of an acoustic event. Fig. 1 shows an example of constructing time difference database in the method.

\section{Wave Velocity of a Transversely Isotropic Medium}

In a transversely isotropic medium, three types of elastic wave are found: compressive wave $\left(V_{P}\right)$, shear wave vibrating parallel to the isotropic plane $\left(V_{S H}\right)$, and shear wave vibrating in a plane perpendicular to the isotropic plane $\left(V_{S V}\right)$. Except for the case when the direction of wave propagation is perpendicular to the isotropic plane, $V_{S H}$ and $V_{S V}$ need distinguishing. Basically, both P-and S- wave are capable of localizing AE events. Amplitude of S-wave is larger than that of Pwave, thus S-wave is less sensitive to the background noise. However, $V_{S H}$ and $V_{S V}$ are difficult to be distinguished by AE sensors used in this study so that P-wave was adopted in the following source locating process.

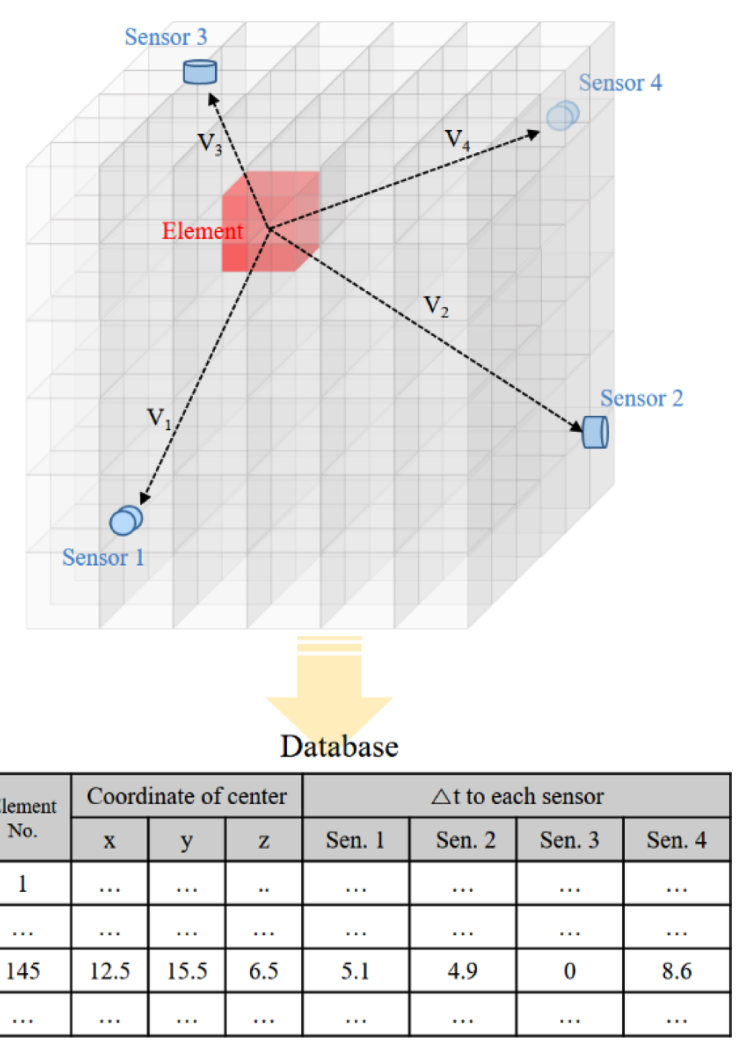

Figure 1. Procedure for constructing time difference database in the partition approximation method. 
Defining an angle between the direction of incident wave and the normal vector of isotropic plane as $\alpha$, the wave velocities are presented in Eq. (1) as a function of the angle $\alpha$ (Daley and Hron, 1977; Song et al., 2004).

$$
\begin{aligned}
& \rho V_{P}^{2}(\alpha)=\frac{1}{2}\left[C_{33}+C_{44}+\left(C_{11}-C_{33}\right) \sin ^{2} \alpha+D(\alpha)\right] \\
& \rho V_{S V}{ }^{2}(\alpha)=\frac{1}{2}\left[C_{33}+C_{44}+\left(C_{11}-C_{33}\right) \sin ^{2} \alpha-D(\alpha)\right] \\
& \rho V_{S H}{ }^{2}(\alpha)=\frac{1}{2}\left(C_{11}-C_{12}\right) \sin ^{2} \alpha+C_{44} \cos ^{2} \alpha \\
& D(\alpha)=\sqrt{\begin{array}{l}
\left(C_{33}-C_{44}\right)^{2}+2\left[2\left(C_{13}+C_{44}\right)^{2}-\left(C_{33}-C_{44}\right)\left(C_{11}+C_{33}-2 C_{44}\right)\right] \\
\sin ^{2} \alpha+\left[\left(C_{11}+C_{33}-2 C_{44}\right)^{2}-4\left(C_{13}+C_{44}\right)^{2}\right] \sin ^{4} \alpha
\end{array}}
\end{aligned}
$$

where $\rho$ is the density of the medium and $D(\alpha)$ is a function of the material properties. Stiffness constants, $C_{i j}$, are calculated by the density and wave velocities under specific angles, as presented in Eq. (2).

$$
\begin{aligned}
& C_{11}=\rho V_{P}^{2}\left(90^{\circ}\right) \quad C_{12}=C_{11}-2 \rho V_{S H}^{2}\left(90^{\circ}\right) \\
& C_{33}=\rho V_{P}^{2}\left(0^{\circ}\right) \quad C_{44}=\rho V_{S H}^{2}\left(0^{\circ}\right)=\rho V_{S V}{ }^{2}\left(0^{\circ}\right)=\rho V_{S V}{ }^{2}\left(90^{\circ}\right) \\
& C_{13}=-C_{44}+\sqrt{\begin{array}{l}
4 \rho^{2} V_{P}^{4}\left(45^{\circ}\right)-\left[2 \rho V_{P}^{2}\left(45^{\circ}\right) \times\left(C_{11}+C_{33}+2 C_{44}\right)\right] \\
+\left[\left(C_{11}+C_{44}\right)\left(C_{33}+C_{44}\right)\right]
\end{array}}
\end{aligned}
$$

Therefore, if one can measure the wave velocities when $\alpha$ equals to $0^{\circ}, 45^{\circ}$, and $90^{\circ}$, the P-wave velocity for any angle $(\alpha)$ can be calculated.

Once geometric parameters, such as the size of specimen, size of element, and the direction of isotropic plane, are determined, the distance from an element to AE sensors and the corresponding angle $(\alpha)$ can be calculated. By plugging $\alpha$ into Eq. (1), the P-wave velocity in the specific direction is obtained and the velocity can be applied fur- ther in the third processing box of the closed loop in Fig. 1. By this procedure, wave velocity anisotropy can be simply taken into account in the partition approximation method.

\section{Arrival Time Picking Algorithm}

A single acoustic event in an experiment emits elastic waves and multiple AE sensors measure those waves. Based on the measured values, the wave arrival time and the time differences in the experiment are calculated, and the location of the event is eventually located by comparing the database and experimental results. Therefore, it is of great importance to precisely measure the wave arrival time in the experiment.

Numerous algorithms for picking wave arrival time have been proposed. Amplitude threshold method is one of the most frequently adopted algorithms because of its simplicity. However, the method has shortcomings. For instance, it is significantly affected by the background noise and prone to provide erroneous results when a threshold value is improperly selected (Kurz et al., 2005). Therefore, several alternatives have been developed to mediate those drawbacks, including AIC (Akaike Information Criterion) (Sleeman and Van Eck, 1999), two-step AIC (Sedlak et al., 2009), and Hinkley pickers (Hinkley, 1971). In this study, the two-step AIC algorithm was used to determine the Pwave arrival time since it gives relatively consistent results regardless of the background noise level (Lee et al., 2011). Some constants in the picking algorithm are affected by the characteristics of the tested material so that trial and error approach was required to set the values.

\section{Overall Source Locating Procedure}

Fig. 2 shows a conceptual example of source locating result adopted in this study. First, a database, which was based on the geometric and

Database by partition approximation method

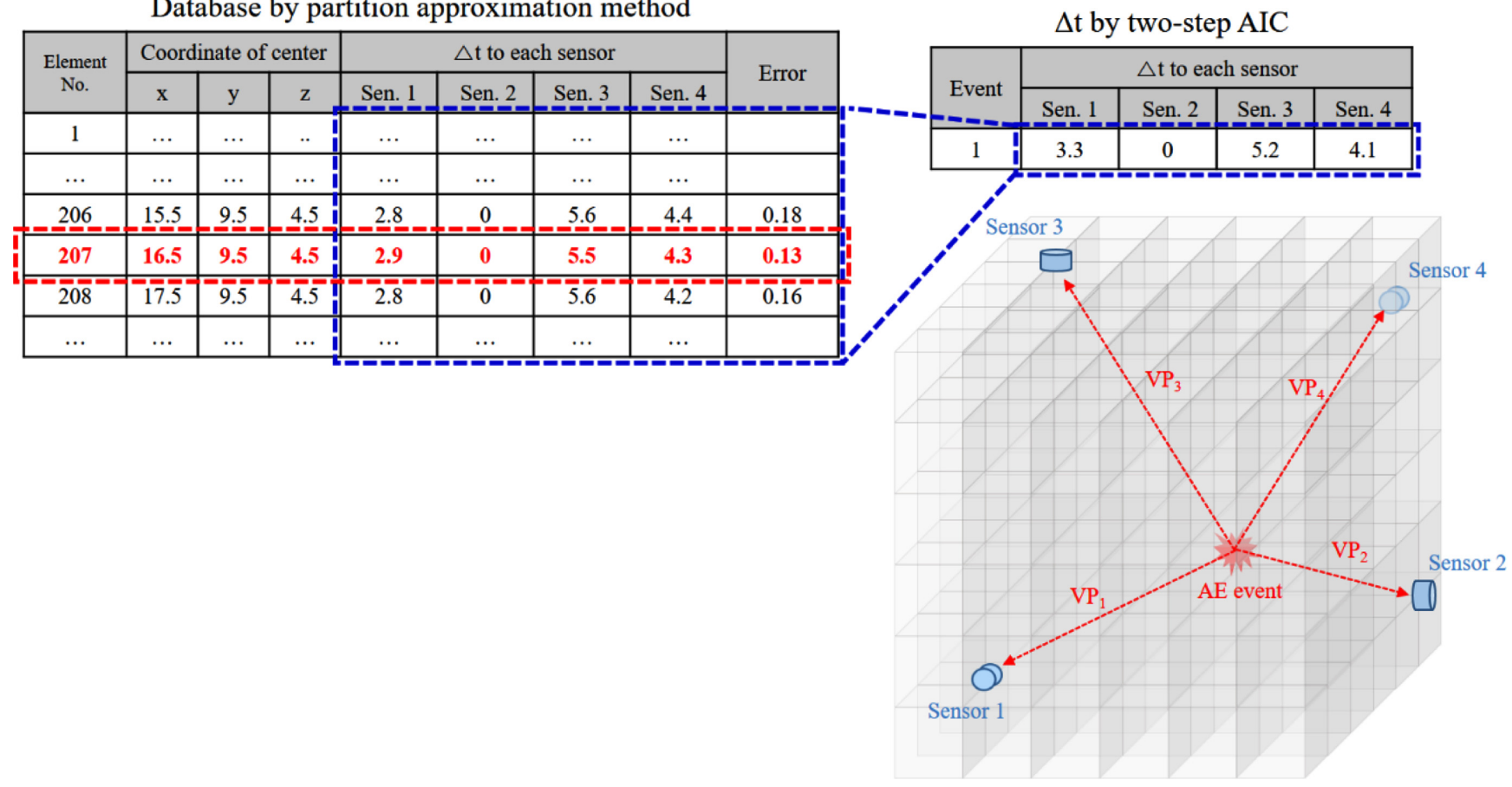

Figure 2. Example of source locating result adopted in this study. 
experimental conditions, such as the size of the specimen and each element, and coordinate of sensors, constructed by the partition approximation method. The database also contained $\Delta t$ values from an element to each $\mathrm{AE}$ sensor and wave velocity anisotropy could be easily taken into account in this part if needed. Besides the database, actual wave arrival time in the experiment was calculated by two-step AIC algorithm. Then, a set of $\Delta t$ values in a measured AE event was compared with sets of $\Delta t$ values in the database. During the comparison, an indicial term was required, for instance the least square in Fig. 2 , to select an element that had the least error. Finally, the coordinates of the element were estimated as the location of $\mathrm{AE}$ event. Though the procedure requires relatively large amount of calculation, the method has several strengths, being straightforward and easy to implement. At the same time, it is applicable for anisotropic cases, which show apparent and inherent stratified structures for example, without solving complicated equations.

\section{Validation Works}

\section{Numerical Calculation on Virtual Cases}

Let us suppose an ideal two-dimensional specimen with three $\mathrm{AE}$ sensors on the periphery. Size of the specimen was set as $100 \times 100 \mathrm{~mm}$.

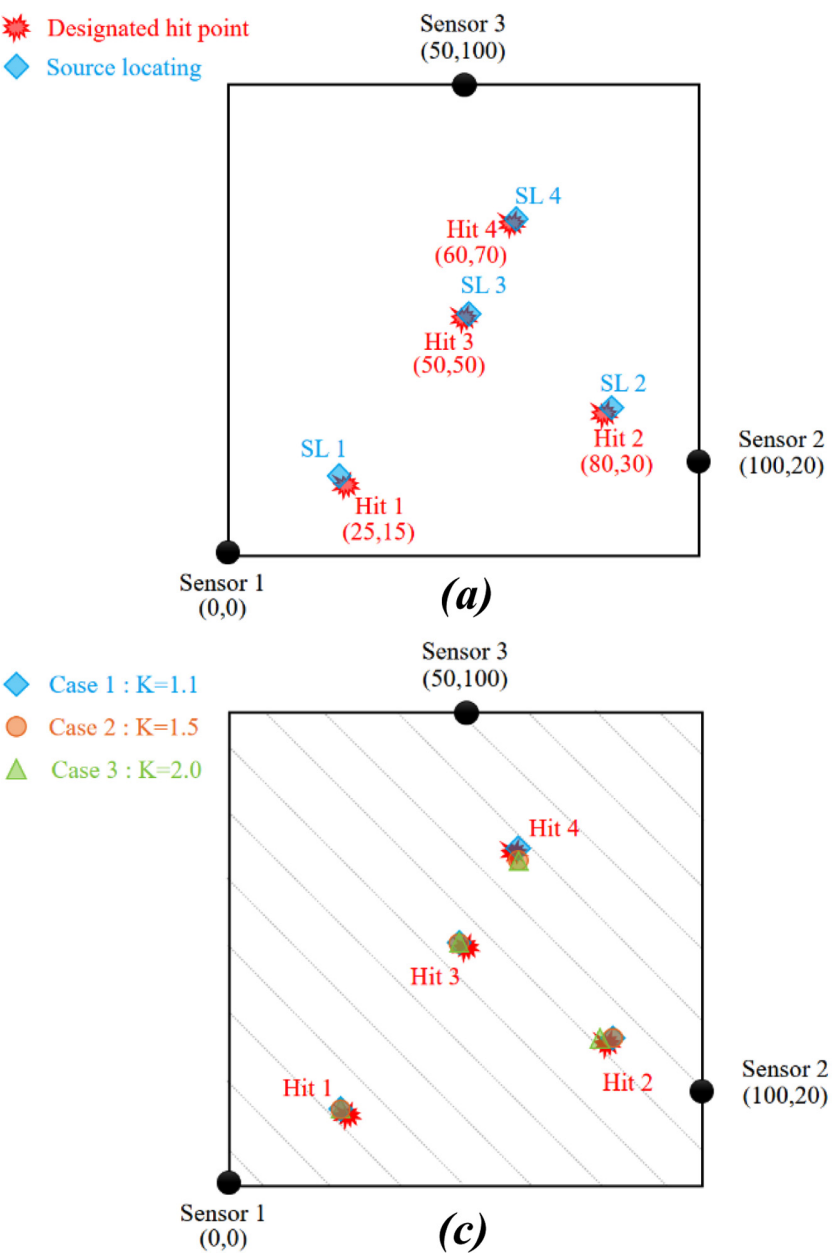

The coordinates of the sensors as well as four virtual hit points are presented in Fig. 3(a). The specimen was considered as isotropic and its P-wave velocity was set as $3,000 \mathrm{~m} / \mathrm{s}$. At the same time, similar circumstances in terms of size, coordinates of sensor and hit were prepared but having clear transversely isotropic structure inside. The angles between the planar structure and horizontal axis were $0^{\circ}, 45^{\circ}$, and $90^{\circ}$, respectively (Fig. 3(b) (d)). In each case, it is apparent that the maximum P-wave velocity would be measured when the wave propagates along parallel direction of planar structure while the minimum would be measured when perpendicular direction. Ratio of maximum over minimum velocity, which was denoted as $\mathrm{K}$ in this study, varied from 1.1, 1.5, and 2.0. Even though the ratio varied, average velocity was set as $3,000 \mathrm{~m} / \mathrm{s}$, which was the same with isotropic case, so the maximum and minimum values of $\mathrm{K}=2.0$ case were 4,000 and 2,000 $\mathrm{m} / \mathrm{s}$, for instance. The whole domain was discretized with the interval of $2.5 \mathrm{~mm}$ so that the number of elements was 1,600. Error of the results was defined as the distance between designated and calculated coordinates (Eq. (3)). The partition approximation method was adopted to all those cases and the results are shown in Fig. 3 and Table 1.

$$
\text { Error }=\sqrt{\left(x-x_{i}\right)^{2}+\left(y-y_{i}\right)^{2}}
$$

Since the domain was partitioned with the interval of $2.5 \mathrm{~mm}$, the example had an inherent resolution of $1.25 \mathrm{~mm}$. In case of the isotro-

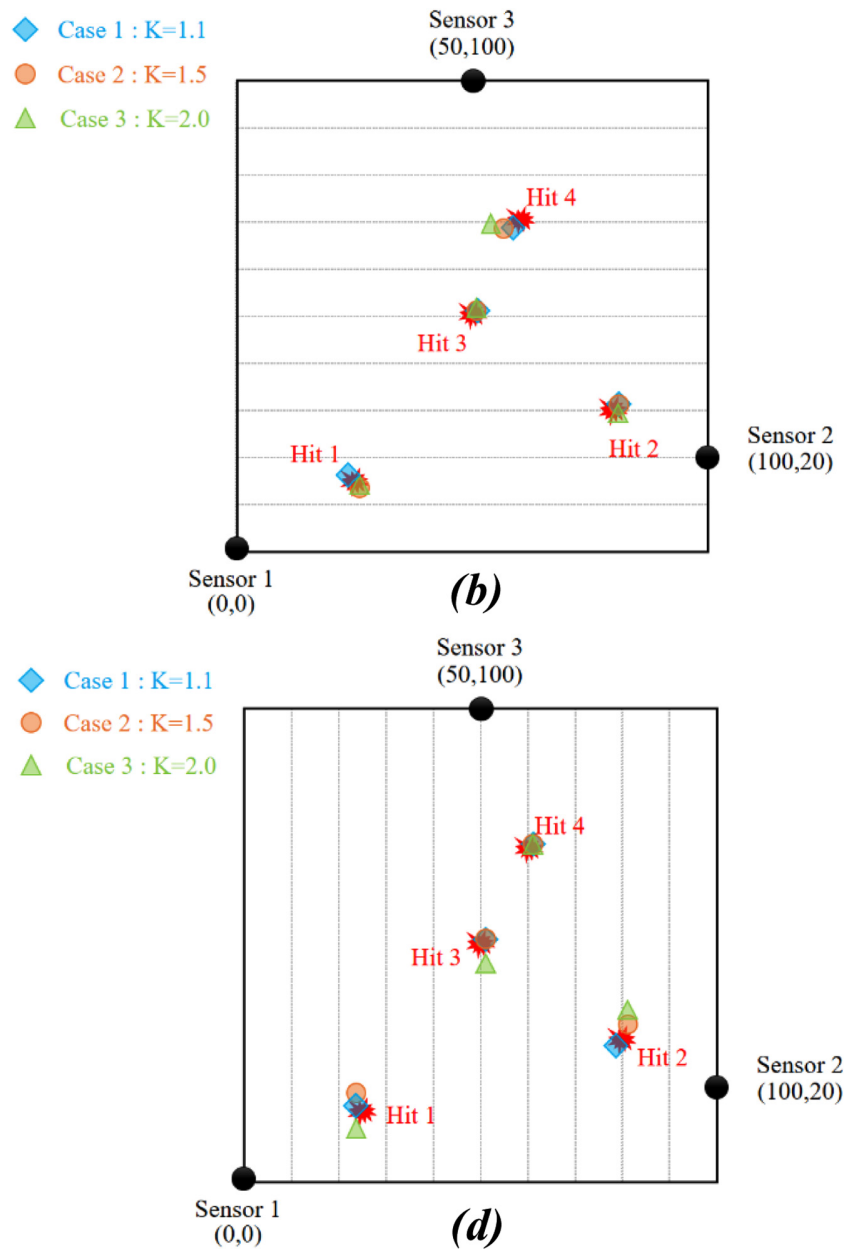

Figure 3. Results of the partition approximation method from each case. (a) isotropic; (b) anisotropic angle of $0^{\circ}$; (c) anisotropic angle of $45^{\circ}$; (d) anisotropic angle of $90^{\circ}$. 
Table 1. Calculated errors of each case based on the partition approximation method

\begin{tabular}{|c|c|c|c|c|c|c|c|c|c|c|}
\hline \multirow{3}{*}{ Hit } & \multirow{3}{*}{ Isotropic } & \multicolumn{9}{|c|}{ Transversely isotropic } \\
\hline & & \multicolumn{3}{|c|}{ Anisotropy angle $=0^{\circ}$} & \multicolumn{3}{|c|}{ Anisotropy angle $=45^{\circ}$} & \multicolumn{3}{|c|}{ Anisotropy angle $=90^{\circ}$} \\
\hline & & $\mathrm{K}=1.1$ & $\mathrm{~K}=1.5$ & $\mathrm{~K}=2.0$ & $\mathrm{~K}=1.1$ & $\mathrm{~K}=1.5$ & $\mathrm{~K}=2.0$ & $\mathrm{~K}=1.1$ & $\mathrm{~K}=1.5$ & $\mathrm{~K}=2.0$ \\
\hline 1 & 1.77 & 1.77 & 1.77 & 1.77 & 1.77 & 1.77 & 1.77 & 1.77 & 3.95 & 3.95 \\
\hline 2 & 1.77 & 1.77 & 1.77 & 1.77 & 1.77 & 1.77 & 1.77 & 1.77 & 3.48 & 6.86 \\
\hline 3 & 1.77 & 1.77 & 1.77 & 1.77 & 1.77 & 1.77 & 1.77 & 1.77 & 1.77 & 3.95 \\
\hline 4 & 1.77 & 1.77 & 3.48 & 6.37 & 1.77 & 1.77 & 1.77 & 1.77 & 1.77 & 1.77 \\
\hline
\end{tabular}

pic case, $1.77 \mathrm{~mm}$ of error value was calculated and it was thought to be acceptable judging from the resolution. With the finer partitioning, smaller error would be expected. In the case of $\mathrm{K}=1.1$ results, which was low degree of anisotropy, the calculated error values were the same with the isotropic cases. In the cases of $\mathrm{K}=1.5$ and 2.0, medium to high degree of anisotropy, the overall errors increased as the $\mathrm{K}$ values increased. The maximum error value was calculated as $6.86 \mathrm{~mm}$ when $\mathrm{K}=2.0$. If the maximum value was normalized by the length of the specimen $(100 \mathrm{~mm})$, approximately $6.9 \%$ of the maximum error was expected by the source locating technique. However, one should be aware that the error might increase in the application on real rock or rock-like material, as the example above was set to on ideal, where

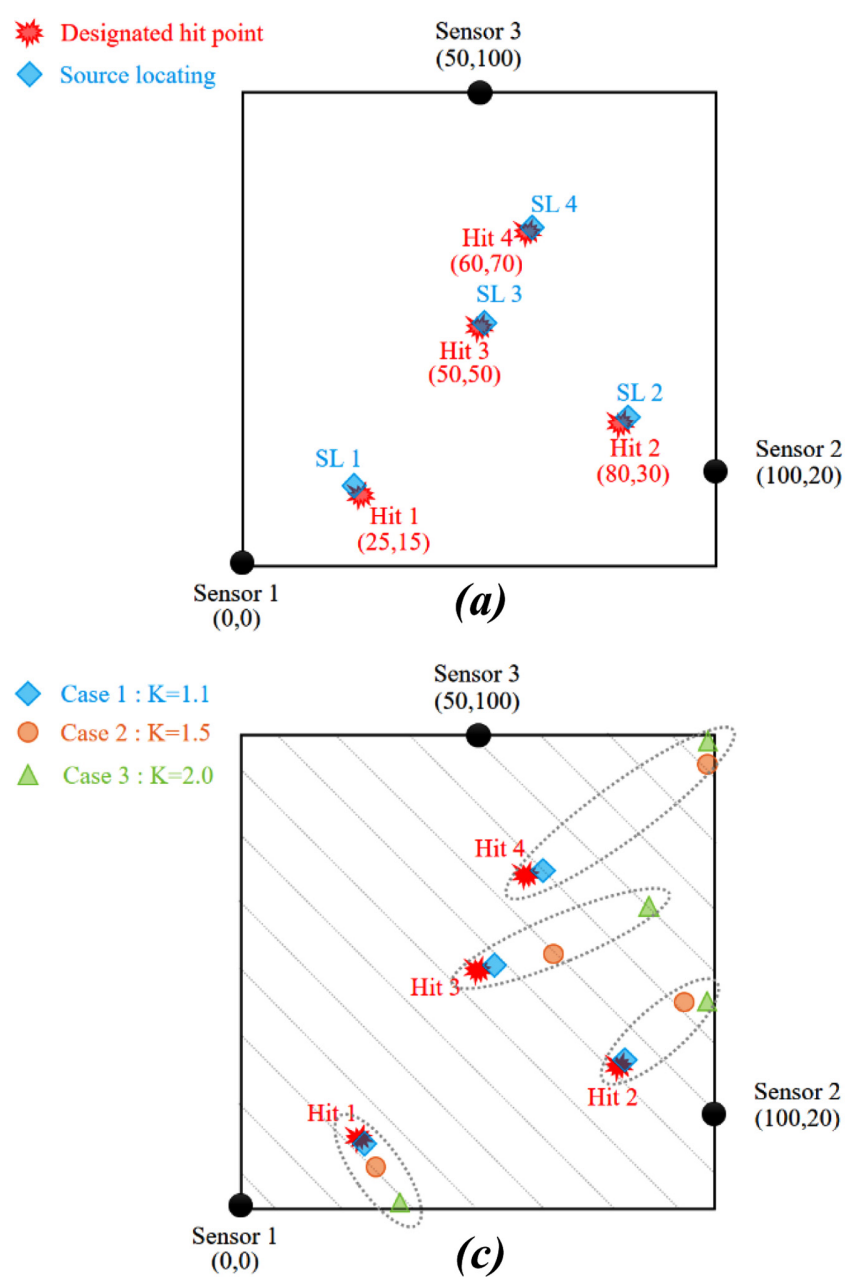

the specimen was continuous and homogeneous.

Meanwhile, Fig. 4 and Table 2 demonstrate the importance of proper treatment about anisotropy. Conditions in terms of size, coordinates of sensors and hit position were the same with the above cases, but the effect of wave anisotropy was not considered in the case. That is, all the cases in Fig. 3 were recalculated with the constant $\mathrm{P}$-wave velocity of $3,000 \mathrm{~m} / \mathrm{s}$, though the anisotropic medium actually had anisotropy ratio of wave velocity (K). Applied wave velocity of 3,000 m/s, corresponded to the average of maximum and minimum velocities of Fig. 3(b) (d) cases.

The results remarkably showed the effect of wave anisotropy. In the case of $\mathrm{K}=1.1$, the calculated errors significantly increased from Fig. 3

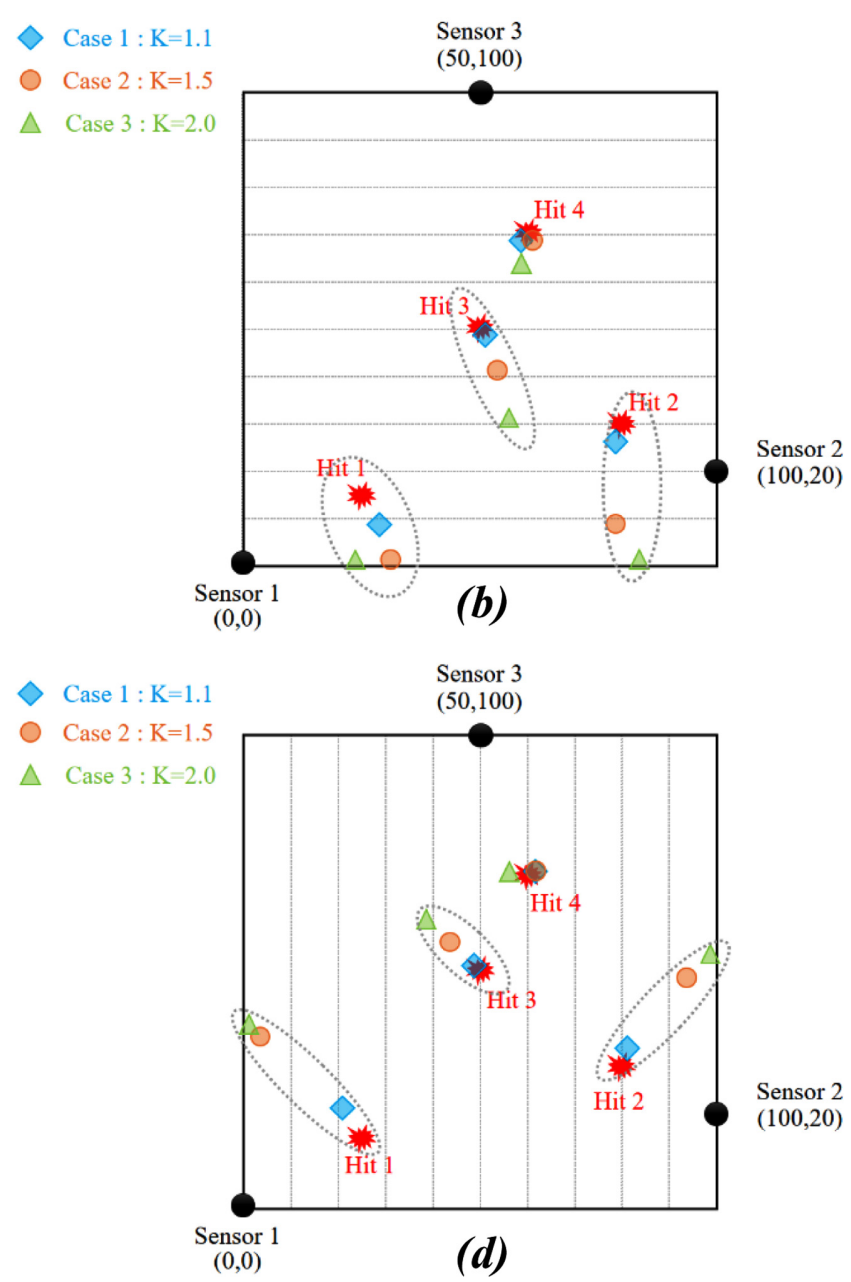

Figure 4. Results of the partition approximation method ignoring the effect of wave anisotropy. (a) isotropic; (b) anisotropic angle of $0^{\circ}$; (c) anisotropic angle of $45^{\circ}$; (d) anisotropic angle of $90^{\circ}$. 
Table 2. Calculated errors of each case ignoring the effect of wave anisotropy

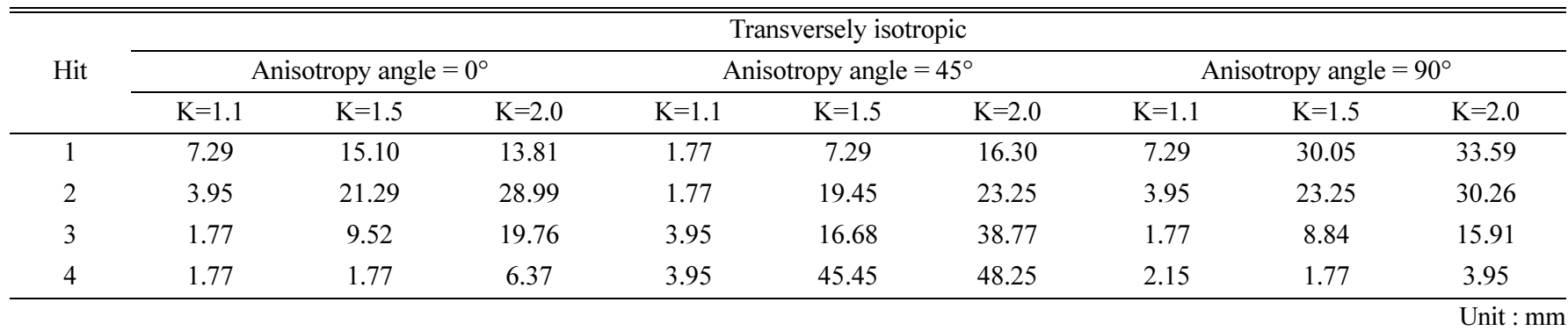

cases, even though the anisotropic ratio was very small. When it comes to the $\mathrm{K}=1.5$ and 2.0 cases, the maximum errors were calculated as 45.45 and $48.25 \mathrm{~mm}$, respectively, which can be regarded as significant. Though the examples above dealt with virtual specimens, anisotropy ratio of wave velocity around 1.1 2.0 could be found in some real rocks. Kim et al. (2012) reported several anisotropic characteristics of typical Korean rocks. Among them, ratios of P-wave anisotropy of Asan gneiss, Boryeong shale, and Yeoncheon schist were roughly 1.1, 1.5 , and 2.3, respectively, which shows that the settings in this study were reasonable. Therefore, it could be concluded that proper consideration of wave anisotropy into AE source locating was of great importance and at the same time, the partition approximation method had several strengths, such as enough applicability, simplicity, and straightforwardness in that consideration.

\section{Pencil Lead Break Test}

In addition to the virtual validation in the previous section, laboratory test, specifically pencil lead break test, was conducted with artificial transversely isotropic specimens. In the test, pencil lead is broken at designated positions to generate $\mathrm{AE}$ events, through which in further elastic waves propagate. Then, multiple AE sensors detect the waves. The wave signals are then analyzed through the partition approximation method so that the location of each event can be calculated. Since the original positions are known, comparison between the original and calculated position can be viable, by which a quantitative validation is made.

The specimens were made of cement mortar so as to minimize the heterogeneity of testing material. They had a remarkable stratified structure inside and each layer was made one by one in sequence, having $10 \mathrm{~mm}$ of thickness. Rectangular parallelepiped specimens (170.0 $\times 171.5 \times 170.0 \mathrm{~mm}$ ) were prepared and tested after five days of curing time at room temperature and humidity. One parallelepiped specimen was used in pencil lead break tests and the others were directionally cored to make cylindrical specimens of $54 \mathrm{~mm}$ in diameter, which met the ISRM suggested method for uniaxial compressive tests in terms of the size and shape (Bieniawski and Bernede, 1979). Since any load was not applied in the tests, $\mathrm{P}$ - and S-wave velocities required in Eq. (1) and (2) were directly measured using the cylindrical specimens for the sake of convenience, and then the velocity profile was calculated based on the measured values. Fig. 5 shows schematic diagrams of the specimens used in this study.

The P-wave velocity increased gradually as the angle theta in Fig. 5 (b) increased. The minimum velocity of $3,996 \mathrm{~m} / \mathrm{s}$ was measured at $\theta=0^{\circ}$ when the direction of wave propagation was perpendicular to the isotropic plane. Whereas the maximum velocity of $4,441 \mathrm{~m} / \mathrm{s}$ at $\theta=90^{\circ}$ was measured when the direction of wave propagation was parallel to the isotropic plane. Anisotropy ratio of the P-wave velocity was calculated to be 1.11, which presented a low level of anisotropy.

The rectangular parallelepiped specimen $(170.0 \times 171.5 \times 170.0 \mathrm{~mm})$ was partitioned into 170 segments along each axis so that total 4,913,000 elements were generated. Each element was approximately cubic of which the center was assumed as a potential source location. The $\mathrm{AE}$ signals were generated by breaking pencil lead at designated positions. The signals were measured by sensors and then the partition approximation method was applied to back-calculate the original hit-
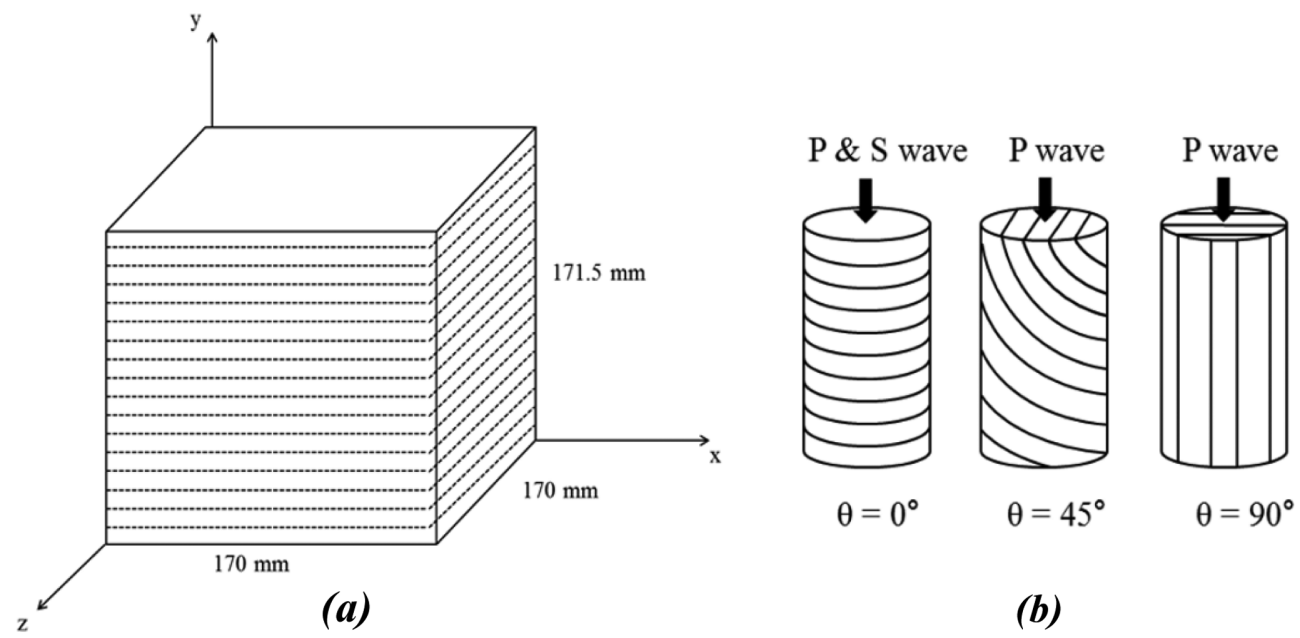

(b)

Figure 5. Schematic diagrams of specimens. (a) rectangular parallelepiped specimen; (b) cylindrical specimens by directional coring. 


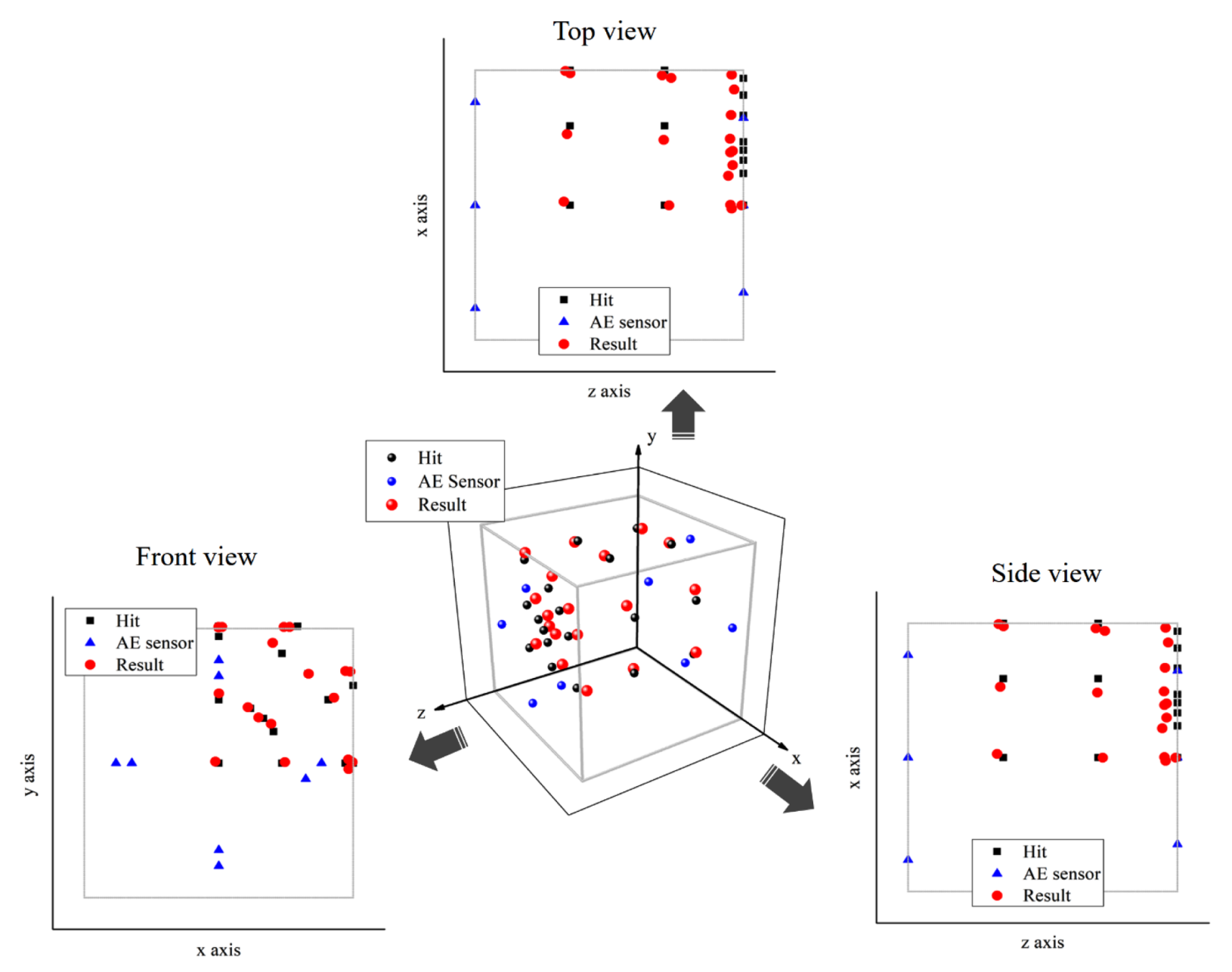

Figure 6. Source locating results from pencil lead break test.

ting positions. Along with the AE sensors, 19 hitting positions were designated, being designed to take various wave propagation angle into account. Fig. 6 shows the source locating results.

Error of the results was defined as the distance between designated and calculated coordinates (Eq. (4)) for quantitative evaluation. All error terms have distance unit, which corresponded to the discrepancies between the hitting positions and back-calculated locations. The results are shown in Table 3.

$$
\text { Error }=\sqrt{\left(x-x_{i}\right)^{2}+\left(y-y_{i}\right)^{2}++\left(z-z_{i}\right)^{2}}
$$

Calculated error varied from 2.88 to $11.22 \mathrm{~mm}$ and its average was $7.46 \mathrm{~mm}$. Considering that the diameter of the AE sensor was 8.25 $\mathrm{mm}$, the results presented the averaged error less than 1D of the sensor. Let us suppose that the specimen was a cube with $170.00 \mathrm{~mm}$ of side length. Then, the calculated errors in Table 3 could be normalized by the length and also be presented as percentages. Average of the normalized error was approximately $4.4 \%$ of the specimen size.

\section{Application}

In order to test the applicability of the partition approximation method further, a series of Brazilian tension tests were carried out on artificial transversely isotropic specimens. In the test, a disk-shaped specimen is loaded diametrically so that macroscopic tensile cracks generate and propagate along the vertical center line of the specimen. Since the specimen contained weak planes inside, which were the boundary between stratified layers, the AE activities could be different from isotropic cases. In this study, Brazilian test was chosen as an application because of its simplicity, relatively well-defined macroscopic cracking behavior, and easiness to observe cracking behavior during the tests.

\section{Experimental Setup}

The specimens used in the Brazilian tension test were made in the same manner for the pencil lead break tests, except for the thickness of the layer that was $5 \mathrm{~mm}$ in this case. Cube specimens with $170 \mathrm{~mm}$ of length were cored in different directions to make the Brazilian disks. The disks had larger dimension of $116.2 \mathrm{~mm}$ in diameter and roughly half-diameter in height than a traditional Brazilian disk (Bieniawski and Hawkes, 1978), which was for clear observation of the cracking behavior. The Brazilian disk had a geometric angle, defined as $\theta$ in this case, which was the dip of the isotropic plane, thus it was anticipated that the cracking behavior was greatly affected by the angle $\theta$. Therefore, a series of Brazilian tests were conducted with varying $\theta$ values from $0^{\circ}$ to $90^{\circ}$ with the interval of $15^{\circ}$.

Eight $\mathrm{AE}$ sensors were used to measure the signals: three on the 
Table 3. Source locating results and their errors

\begin{tabular}{|c|c|c|c|c|c|c|c|}
\hline \multirow{2}{*}{ Hit num. } & \multicolumn{3}{|c|}{ Designated hit position } & \multicolumn{3}{|c|}{ Calculated result } & \multirow{2}{*}{ Error } \\
\hline & $\mathrm{x}$ & $\mathrm{y}$ & $\mathrm{Z}$ & $\mathrm{x}$ & $\mathrm{y}$ & $\mathrm{Z}$ & \\
\hline 1 & 85.00 & 85.00 & 170.00 & 82.83 & 85.92 & 162.50 & 7.86 \\
\hline 2 & 125.00 & 85.00 & 170.00 & 126.83 & 85.58 & 161.50 & 8.71 \\
\hline 3 & 165.00 & 85.00 & 170.00 & 167.16 & 81.21 & 162.50 & 8.68 \\
\hline 4 & 119.64 & 105.00 & 170.00 & 118.16 & 109.82 & 161.83 & 9.60 \\
\hline 6 & 113.28 & 113.28 & 170.00 & 110.16 & 113.83 & 163.17 & 7.53 \\
\hline 7 & 141.57 & 141.57 & 170.00 & 141.83 & 141.40 & 162.17 & 7.84 \\
\hline 8 & 105.00 & 119.64 & 170.00 & 103.50 & 120.31 & 160.50 & 9.64 \\
\hline 9 & 125.00 & 154.28 & 170.00 & 119.16 & 161.00 & 163.17 & 11.22 \\
\hline 10 & 85.00 & 125.00 & 170.00 & 85.16 & 129.04 & 161.83 & 9.12 \\
\hline 12 & 170.00 & 85.00 & 120.00 & 166.83 & 87.26 & 118.50 & 4.17 \\
\hline 13 & 170.00 & 85.00 & 60.00 & 169.50 & 85.58 & 57.17 & 2.93 \\
\hline 14 & 170.00 & 134.07 & 120.00 & 165.16 & 143.19 & 124.17 & 11.14 \\
\hline 15 & 170.00 & 134.07 & 60.00 & 168.16 & 142.90 & 60.17 & 9.02 \\
\hline 16 & 134.94 & 171.50 & 120.00 & 126.16 & 171.00 & 119.50 & 8.81 \\
\hline 17 & 134.94 & 171.50 & 60.00 & 129.83 & 171.00 & 58.17 & 5.45 \\
\hline 18 & 85.00 & 171.50 & 120.00 & 84.83 & 171.00 & 122.83 & 2.88 \\
\hline 19 & 85.00 & 171.50 & 60.00 & 87.16 & 171.00 & 56.27 & 4.34 \\
\hline
\end{tabular}

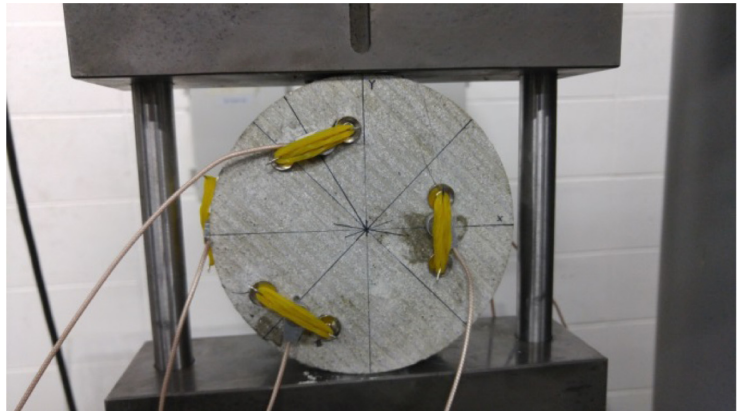

(a)

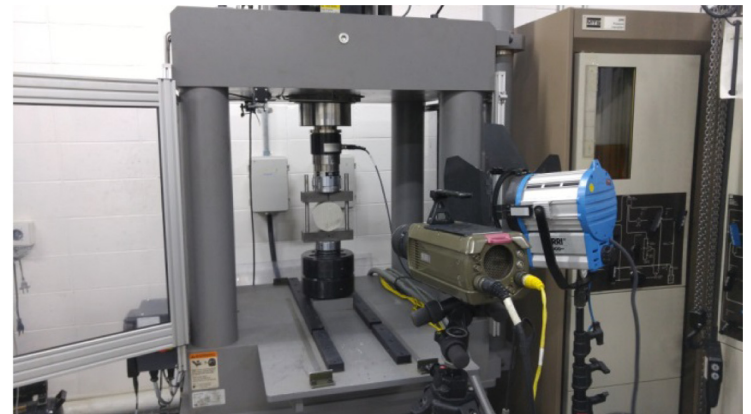

(b)

Figure 7. Experimental setup for Brazilian tension test. (a) specimen and sensor arrangement; (b) overall setup.

front surface, three on the back surface, and two on the middle of the cylinder side walls. Defining the origin of the coordinates system as the center point of the back surface, the position of AE sensors was fixed, whereas the angle $\theta$ varied. Fig. 7 shows the experimental setup. During the Brazilian tension tests, a high speed camera (20,000 frames per second) recorded the cracking behavior, focusing on the front surface of the disks.

\section{Results and Discussion}

The maximum P-wave velocity was measured as $4,548 \mathrm{~m} / \mathrm{s}$ and the minimum velocity as $4,107 \mathrm{~m} / \mathrm{s}$ from cylindrical specimen so that the wave anisotropy ratio was 1.11 . It is known that not only the natural anisotropic feature of rocks, but stress could induce wave anisotropy since it compresses or generates cracks depending on the stress state (Nur and Simmons, 1969; Yanagidani et al., 1985; Stanchits et al., 2006).
Stress-induced crack as well as inherent feature made it difficult to figure out variation of wave field. Tensile strength of tested material was not high (maximum $3.25 \mathrm{MPa}$ and minimum 1.31 MPa when $\theta$ equaled $0^{\circ}$ and $90^{\circ}$, respectively) so that the variation of wave velocity was assumed to be small. At the same time, majority of AE count, energy, and detected cracks were measured within last $10 \%$ of stress level, during the very short period as shown in Fig. 8. In Fig. 8, stress level means normalized stress state by its failure strength and percentage of cracks denotes normalized number of cracks by total number of source locating results under specific stress state. Thus, the stress-induced variation of wave velocity was not considered in this study.

For an isotropic medium, typical cracking normally takes place along the vertical center line in the Brazilian test. However, for a transversely isotropic medium, cracks deviated from the center line and secondary cracks were observed quite often. In addition, shear sliding and/or splitting occurred in some cases. Fig. 9 shows an example of high speed camera recording when $\theta=30^{\circ}$ specimen was tested. 


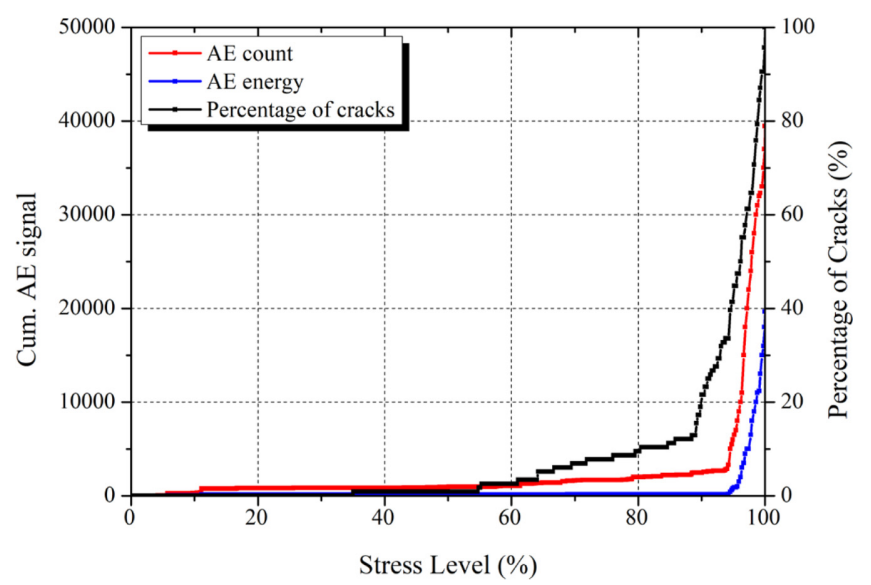

Figure 8. Measured AE activities during Brazilian tensile test $\left(\theta=45^{\circ}\right.$ case).

For clear understanding, red lines were drawn in each snapshot, which illustrates the visible failures on the front surface. As it can be seen, a secondary crack along the layer boundaries was observed then it propagated toward the center of disk due to bending effect. Also, macroscopic and visible cracking behavior took place within $3 \mathrm{~ms}$, which implicated similar results with Fig. 8.

Fig. 10 shows the AE source locating results and high speed camera recordings. Source locating results in Fig. 10 are the projections of all sources of AE events to the front surface. Red lines indicate ultimate failure line captured from camera snapshots. The capacity problem of high speed camera made synchronization between $\mathrm{AE}$ and high speed camera difficult so that the results could not be grouped according to time or loading phase. Hence, Fig. 10 shows the ultimate failure line and all $\mathrm{AE}$ events recorded throughout a test.

AE source locating results were somewhat scattered due to some reasons; tensile cracks make small energy compared to shear cracks or pencil lead break so that it was difficult to pick arrival time exactly through the useful two-step AIC picking method. The tested volume was small and there might be some wave reflection in the specimen boundary, which could affect the waveform and the arrival time to the sensors. This might also affect the accuracy of the partition approximation method. In order to improve the accuracy and precision of source locations, more sensors were desirable but it was not easy considering the size of specimen. With this limitation, the cracking behavior and $\mathrm{AE}$ source locating results were compared and analyzed.

When the angle $\theta$ was less than $30^{\circ}$ (Fig. 10(a) (c)), the effect of anisotropy on cracking behavior was not significant so that the failure lines were formed relatively at the center or vicinity of the center. However, secondary cracks were observed at around the loading part and additional failure due to bending was observed. Judging from the ultimate failure line, the effects of anisotropy could be observed clearly when the $\theta$ was larger than $45^{\circ}$ (Fig. 10(d) (g)) . Failure occurred not at the center but at somewhat deviated regions. At some parts, remarkable shear sliding and secondary cracks were observed as well. The cracks were thought to be generated by tensile stress, however, some of them were located on the boundary of layers. Especially in Fig. 10(e) and (g), the failure line followed the boundaries of weak plane, which indicated the possible effects of shear sliding or splitting.

This trend was confirmed clearly when considering the variation of tensile strength. The tensile strength, nominal strength in this case, can be calculated by Eq. (5).

$$
\sigma_{t}=\frac{2 P}{\pi D t}
$$

where $P$ is the load at failure, $D$ and $t$ are diameter and thickness of the specimen, respectively. Since the strength did not take anisotropy into account, it was nominal or apparent. Fig. 11 shows the variation of the strength at different values of $\theta$.

As described earlier, the effect of anisotropy became remarkable even in terms of the strength. When $\theta$ was larger than $45^{\circ}$, differences in the failure pattern, i.e. the deviation from the center line, could be explained by the decrease in strength. Also, even though the deviations were not large in the case of Fig. 10(e) and (g), the significant decrease in strength indicated that the failure occurred due to shear sliding or splitting.

Observed failure pattern was comparable with the experimental result of Tavallali and Vervoort (2010). They conducted a series of Brazilian tension tests with layered sandstone. They reported that transition in failure mode from central fracture to layer activation, which corresponded with the effect of anisotropy, was in the range of $45^{\circ}$ and $60^{\circ}$. And the latter became dominant when $\theta$ was larger than $60^{\circ}$. Experimental results of this study, showed good agreement with them.

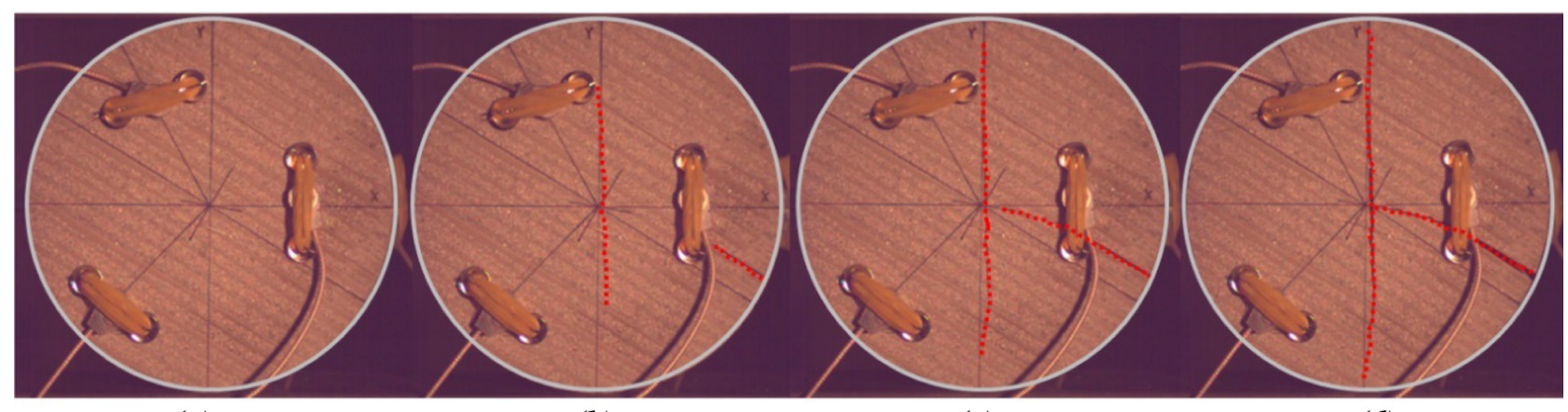

(a)

(b)

(c)

(d)

Figure 9. Macroscopic cracking behavior recorded by high speed camera $\left(\theta=30^{\circ}\right.$ case). (a) $t=0 \mathrm{~ms}$ (triggered); (b) $t=0.90 \mathrm{~ms}$; (c) $t=1.35 \mathrm{~ms}$; (d) $t=2.95 \mathrm{~ms}$. 


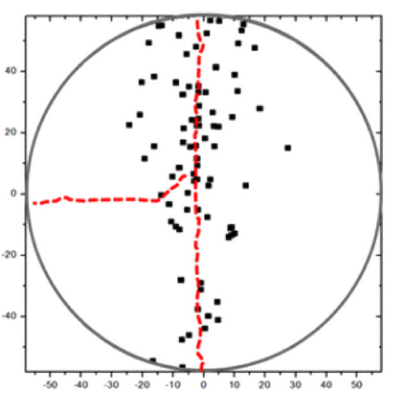

(a) $\theta=0^{\circ}$
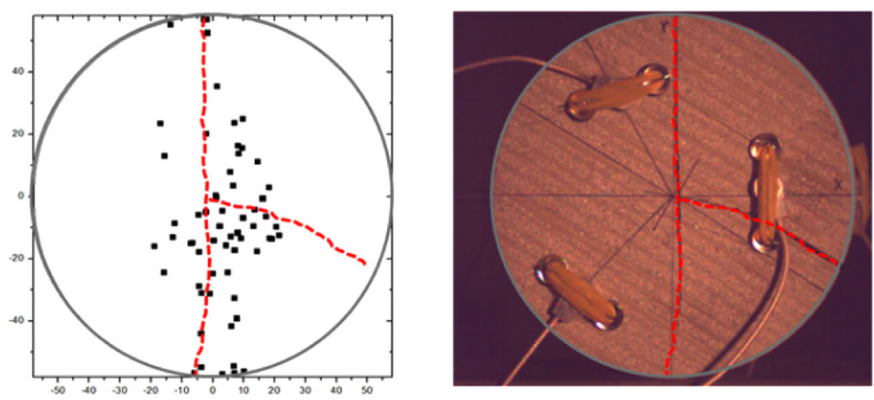

(c) $\theta=30^{\circ}$
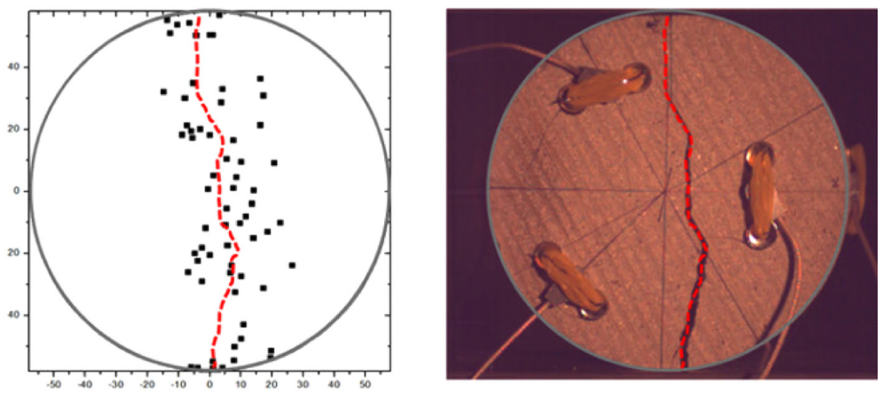

(e) $\theta=60^{\circ}$
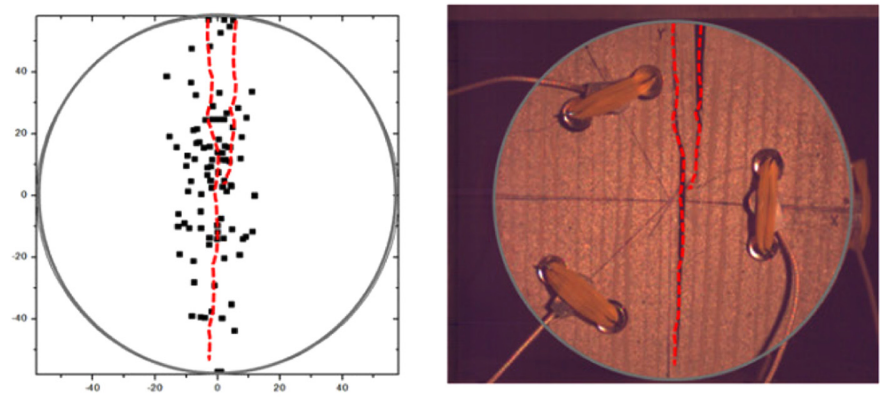

(g) $\theta=90^{\circ}$

Figure 10. Source locating results and snapshots at ultimate failure.

\section{Conclusions}

Acoustic emission (AE), which is generated from microfracturing in a solid, is useful in detection of cracking behavior under various cases. Source locating means a procedure that localizes AE event based on the wave velocity through a medium and measured time differences between AE sensors. Several source locating techniques have been proposed and among them, partition approximation method and its applicability was tested in this study. It partitions a medium into small elements and establishes a database of time differences between
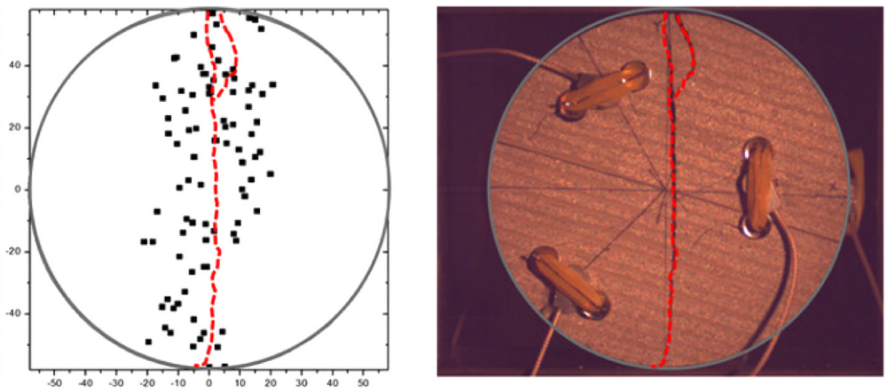

(b) $\theta=15^{\circ}$
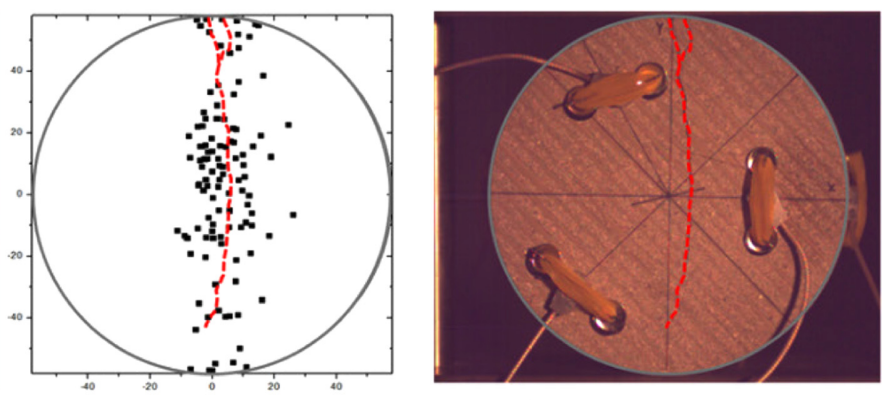

(d) $\theta=45^{\circ}$
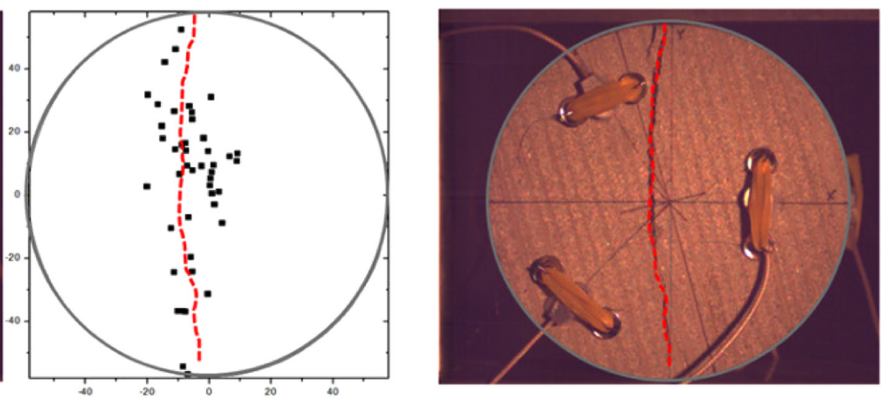

(f) $\theta=75^{\circ}$

the elements and AE sensors. The method has many strengths, such as simplicity, straightforwardness, and easiness in taking anisotropic feature of medium into account.

Validation works through numeric calculation were conducted. Using ideal, two-dimensional specimen, it was shown that the method was capable of being adopted in both isotropic and transversely isotropic media, with maximum $6.86 \mathrm{~mm}$ of calculation error. At the same time, the importance of proper treatment on wave anisotropy was highlighted through the partition approximation method. Unless considering the wave velocity anisotropy, the calculation error became significant, as the ratio of maximum to minimum wave velocity increased. 


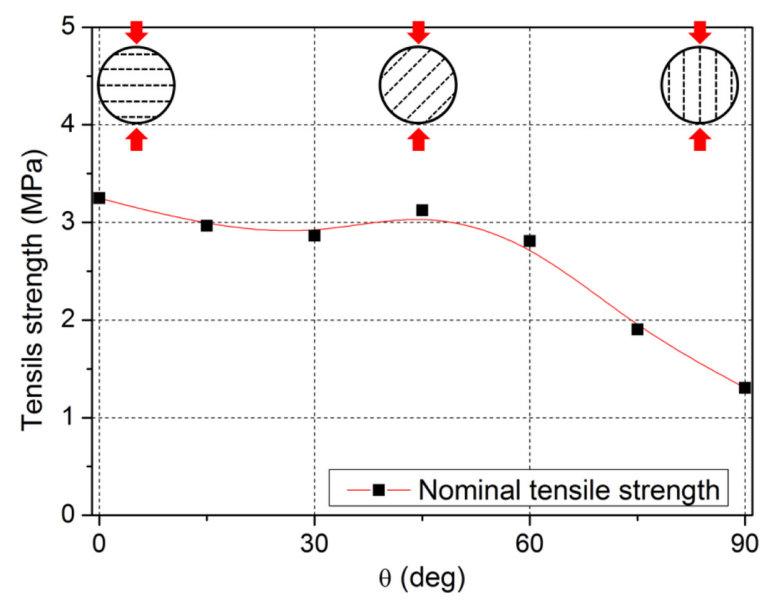

Figure 11. Variation of the nominal tensile strength at different angle of $\theta$.

Pencil lead break tests were carried out as an experimental validation as well. Artificial transversely isotropic specimens were prepared, which were made of cement mortar showing clear stratified structure inside, and used in a series of validation tests. AE signals were generated by breaking pencil lead at designated positions and measured by multiple AE sensors. As a result, quantitative evaluation was conducted by comparing the coordinates of original hit points and backcalculated coordinates. Calculated error varied from 2.88 to $11.22 \mathrm{~mm}$ with the average of $7.46 \mathrm{~mm}$. Despite potential heterogeneity of the specimen, averaged error was less than the diameter of AE sensors. At the same time, if the error were normalized by the length of specimen, approximately $4.4 \%$ of the length was estimated as error in average.

A series of Brazilian tension tests were conducted as an application to observe cracking behavior by $\mathrm{AE}$ measurement and high speed camera recording. Brazilian disks made of cement mortar showed clear anisotropic behavior in terms of failure pattern. The pattern and failure line clearly showed the effect of anisotropy, such as shear sliding and splitting along layer boundaries. The majority of AE events were measured at the last stage of loading, after $90 \%$ of failure stress, so that the stress-induced wave anisotropy was not included in the results. The comparisons between the failure snapshot and source locating results showed that the technique was applicable to observe the cracking behavior of transversely isotropic cases to some extent.

\section{Acknowledgement}

This research was financially supported by the Basic Research and Development of the Korean Institute of Geoscience and Mineral Resources (20-3115) and the Technology Advancement under the Ministry of Land, Infrastructure and Transport in Korea (Project no.: 20CTAP-C152888-02). The Institute of Engineering Research at Seoul National University (SNU) provided research facilities for this work. The authors are grateful for the support.

\section{References}

Bieniawski, Z., and Bernede, M., 1979, Suggested methods for determining the uniaxial compressive strength and deformability of rock mate- rials. International Journal of Rock Mechanics and Mining Science and Geomechanics, Abstracts, v. 16, pp. 138-140.

Bieniawski, Z., and Hawkes, I., 1978, Suggested methods for determining tensile strength of rock materials. International Journal of Rock Mechanics and Mining Sciences and Geomechanics, Abstracts, v. 15, pp. 99-103.

Chang, S., 2002, Characterization of stress-induced damage in rock and its application on the analysis of rock damage zone around a deep tunnel. $\mathrm{Ph}$. D. thesis, Seoul National University, Seoul, Korea, pp. 18-22. (In Korean)

Daley, P.F., and Hron, F., 1977, Reflection and transmission coefficients for transversely isotropic media. Bulletin of the Seismological Society of America, v. 67, pp. 661-675.

Ebrahimkhanlou, A., and Salamone, S., 2017, Acoustic emission source localization in thin metallic plates: A single-sensor approach based on multimodal edge reflections. Ultrasonics, v. 78, pp. 134-145.

Grosse, C., Reinhardt, H., and Dahm, T., 1997, Localization and classification of fracture types in concrete with quantitative acoustic emission measurement techniques. NDT \& E International, v. 30, pp. 223-230.

Hinkley, D., 1971, Inference about the change-point from cumulative sum tests, Biometrika, v. 58, pp. 509-523.

Huang, J., Flynn, J., Mustonen, S., and Young, R., 2016, 3D seismic imaging for monitoring rock-mass degradation and excavation damage. Proceedings of the 2nd International Conference on Rock Dynamics and Applications, pp. 301-307.

Ishida, T., 1999, An introduction to acoustic emission of rock. Kinmiraisha Nagoya, Tokyo, $213 \mathrm{p}$.

Ishida, T., Labuz, J.F., Manthei, G., Meredith, P.G., Nasseri, M.H.B., Shin, K., Yokoyama, T., and Zang, A., 2017, ISRM suggested method for laboratory acoustic emission monitoring. Rock Mechanics and Rock Engineering, v. 50, pp. 665-674.

Kim, H., Cho, J.W., Song, I., and Min, K.B., 2012, Anisotropy of elastic moduli, P-wave velocities, and thermal conductivities of Asan gneiss, Boryeong shale, and Yeoncheon schist in Korea. Engineering Geology, v. 147-148, pp. 68-77.

Kim, S.J., Kwon, S.K., Sanchez, M., and Cho, G.C., 2011, Geological storage of high level nuclear waste. KSCE Journal of Civil Engineering, v. 15 , pp. 721-737.

Kurz, J.H., Grosse, C., and Reinhardt, H., 2005, Strategies for reliable automatic onset time picking of acoustic emission and of ultrasound signals in concrete. Ultrasonics, v. 43, pp. 538-546.

Labuz, J.F., Cattaneo, S., and Chen, L., 2001, Acoustic emission at failure in quasi-brittle materials. Construction and Building Materials, v. 15, pp. 225-233.

Lee, K.S., Kim, J.S., Lee, C.S., Yoon, C.H., and Choi, J.W., 2011, A study on the $\mathrm{P}$ wave arrival time determination algorithm of acoustic emission (AE) suitable for $P$ waves with low signal-to-noise ratios. Tunnel \& Underground Space, v. 21, pp. 349-358. (in Korean)

Maji, A.K., Ouyang, C., and Shah, S.P., 1990, Fracture mechanisms of quasi-brittle materials based on acoustic emission. Journal of Material Research, v. 5, pp. 206-217.

Nur, A., and Simmons, G., 1969, Stress-induced velocity anisotropy in rock: an experimental study, Journal of Geophysical Research, v. 74, pp. 6667-6674.

Ohno, K., and Ohtsu, M., 2010, Crack classification in concrete based on acoustic emission. Construction and Building Materials, v. 24, pp. 2339-2346.

Ohtsu, M., 1995, Acoustic emission theory for moment tensor analysis. Journal of Research in Nondestructive Evaluation, v. 6, pp. 169-184.

Olsson, O., and Winberg, A., 1996, A current understanding of extent and properties of the excavation disturbed zone and its dependence of excavation method. Proceedings of International Conference on Deep Geological Disposal of Radioactive Waste, pp. 101-112.

Ouyang, C., Landis, E., and Shah, S.P., 1991, Damage assessment in concrete using quantitative acoustic emission. Journal of Engineering Mechanics, v. 117, pp. 2681-2698. 
Scruby, C.B., Baldwin, G.R., and Stacey, K.A., 1985, Characterization of fatigue crack extension by quantitative acoustic emission. International Journal of Fracture, v. 28, pp. 201-222.

Sedlak, P., Hirose, Y., Khan, S., Enoki, M., and Sikula, J., 2009, New automatic localization technique of acoustic emission signals in thin metal plate. Ultrasonics, v. 49, pp. 254-262.

Sleeman, R., and Van Eck, T., 1999, Robust automatic P-phase pickings: an on-line implementation in the analysis of broadband seismogram recordings. Physics of the Earth and Planetary Interiors, v. 113, pp. 265-275.

Song, I., Suh, M., Woo, Y., and Hao, T., 2004, Determination of the elastic modulus set of foliated rocks from ultrasonic velocity measurements. Engineering Geology, v. 72, pp. 293-308.

Soulioti, D., Barkoula, N.M., Paipetis, A., Matikas, T.E., Shiotani, T., and Aggelis, D.G., 2009, Acoustic emission behavior of steel fibre rein-

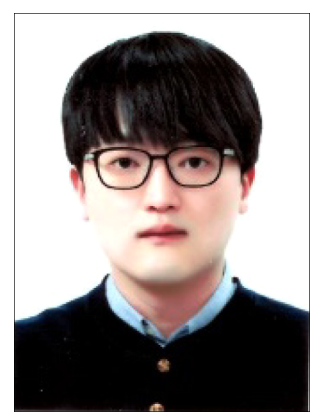

Seungbeom Choi is a researcher of the geology division in Korea Institute of Geoscience and Mineral Resources, Korea. He received his $\mathrm{Ph} . \mathrm{D}$. degree from department of energy resources, Seoul National University, Korea. His research interests mainly focus on rock mechanics and engineering geology field, specifically on hydraulic behavior in rock mass and acoustic emission.

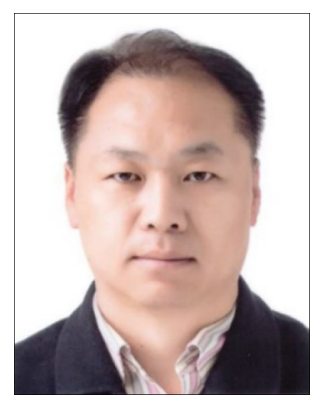

Dae-sung Cheon is a principal researcher in the Geology Division of Korea Institute of Geoscience and Mineral Resources (KIGAM). He received his B. A. (1997), and M. Sc. (1999) in the department of Mineral and Petroleum Engineering of Seoul National University, and Ph.D. (2006) in School of Urban, Civil and Geosystem Engineering from Seoul National University, Korea. He was the research scholar of Pennsylvania State University in USA (2009 2010) and continuing collaboration with Pennsylvania State University. He is interested in the experimental \& the numerical stability analysis of rock structures and monitoring of rock structures using acoustic emission and microseismic technics. forced concrete under bending. Construction and Building Materials, v. 23, pp. 3532-3536.

Stanchits, S., Vinciguerra, S., and Dresen, G., 2006, Ultrasonic velocities, acoustic emission characteristics and crack damage of basalt and granite. Pure and Applied Geophysics, v. 163, pp. 975-994.

Tavallali, A., and Vervoort, A., 2010, Effect of layer orientation on the failure of layered sandstone under Brazilian test conditions. International Journal of Rock Mechanics and Mining Science, v. 47, pp. 313-322.

Yanagidani, T., Ehara, S., Nishizawa, O., Kusunose, K., and Terada, M., 1985, Localization of dilatancy in Oshima granite under constant uniaxial stress. Journal of Geophysical Research, v. 90, pp. 6840-6858.

Yin, S., Cui, Z., and Kundu, T., 2018, Acoustic source localization in anisotropic plates with " $Z$ " shaped sensor clusters. Ultrasonics, v. 84, pp. 34-37.

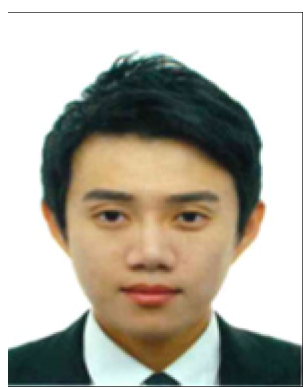

Hoyoung Jeong is a researcher of the Department of energy resources engineering in Seoul National University, Korea. He received his $\mathrm{Ph} . \mathrm{D}$. degree in rock mechanics from the Seoul National University. His research interest includes rock mechanics, mechanized tunneling, rock cutting, and rock fragmentation.

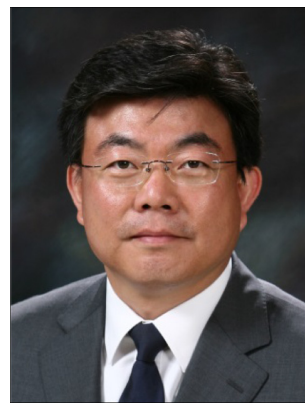

Seokwon Jeon is a professor of the Department of Energy Resources Engineering at Seoul National University, Korea. He received his Ph.D. degree from the University of Arizona in 1996. He published more than 200 journal articles. Currently he is President of Korean Society for Rock Mechanics and Rock Engineering. He has served as VicePresident for Asian of International Society for Rock Mechanics and Rock Engineering for the office term 2015-2019. His current research interests include rock fragmentation by mechanical cutting and explosive blasting. 